\title{
High Resolution Transmission Electron Microscopy and Selected Area Diffraction Study of Doped Zinc Oxide Thin Film
}

\author{
L. Fang, P. Ricou, and R. Korotkov
}

Arkema Inc. 900 First Avenue, King of Prussia, PA 19406

Poly-crystalline Transparent Conductive Oxides such as $\mathrm{ZnO}$ have wide applications in modern electronics, such as the back electrodes in Light Emitting Diodes, Field-effect Transistor Controlled Liquid Crystal Displays, as well as Photovoltaic Solar Panels [1]. In order to lower their sheet resistance and boost their performance, fine tuning of their poly-crystalline structures and doping profile may be necessary. Understanding crystalline structure of the grains can assist in making improvements to the coating performance such as the electronic conductivity, since the grain size and structure can affect the carrier mobility significantly $[2,3]$.

High Resolution Transmission Electron Microscopy analysis was conducted on doped $\mathrm{ZnO}$ deposited by atmospheric pressure chemical vapor deposition at elevated temperatures. The TEM images and the Selected Area Diffraction (SAD) patterns suggested that the CVD growth process followed the so-called "competitive growth" mechanism and results in columnar $\mathrm{ZnO}$ structure. Inexplicably, it appears that there are small inclusions of the crystals below the $\mathrm{ZnO}$-glass interface (Fig. 1). These crystals have a typical size of 3-7 nm in diameter. Immediately after that, when the crystal starts their growth from the glass substrate, the crystal size is small $(5-10 \mathrm{~nm})$, and the diffraction pattern shows multi-orientation poly-crystalline characteristics.

With the increasing thickness of the growing layers, the crystal grain size is increasing, reaching $30 \sim 100 \mathrm{~nm}$ in diameter for $500 \mathrm{~nm}$ thick film at the final stage of the crystal growth (Fig. 2). Due to shadowing of the slow growing crystal planes by the faster growing and more thermodynamically stable ones, a single crystalline orientation (002) becomes a predominate one. SAD patterns show more single-crystalline characteristics in these regions that correlate well with electrical properties of the top regions of the coatings. The diffraction pattern showed strong diffraction spots that originate from (002), (100), (101), (102) and (103) crystal planes. The zone axis along the e-beam was identified to be the $[0,2,0]$ direction. 


\section{References}

[1] Transparent conductive zinc oxide, K. Ellmer editor, Springer, 2008.

[2] Y. Jin et al., JVST A - Vacuum, Surfaces, and Films 15(1997), 1103- 1107.

[3] M. Birkholz et al. Phys. Rev., 68(2003) 205414

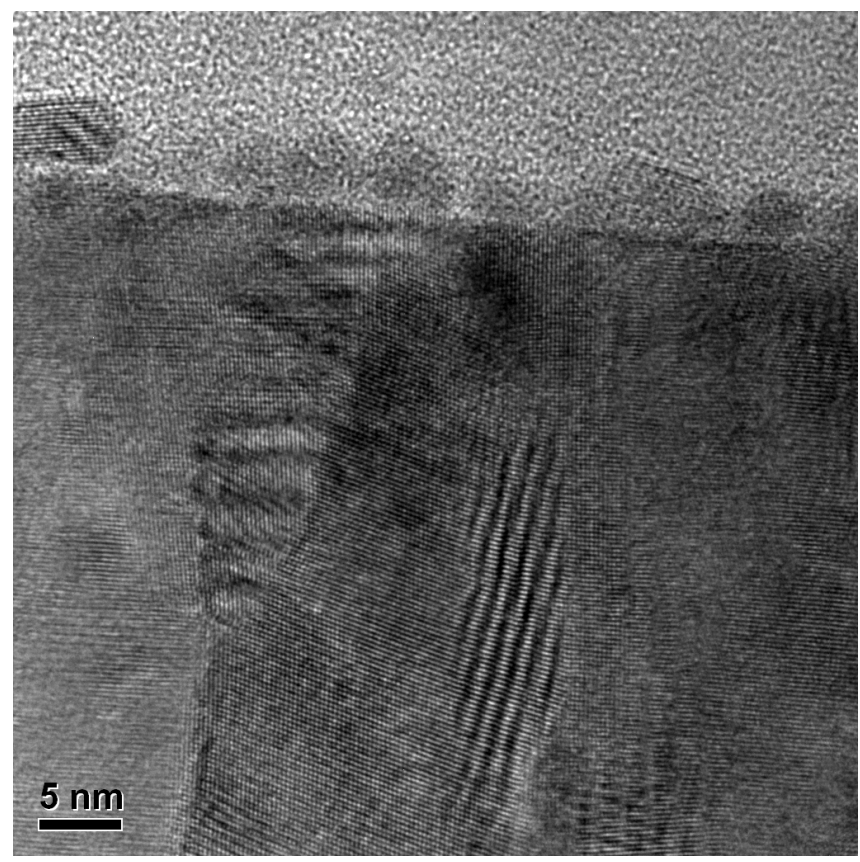

Fig. 1. Initial stage of crystal growth results in small $\mathrm{ZnO}$ columns

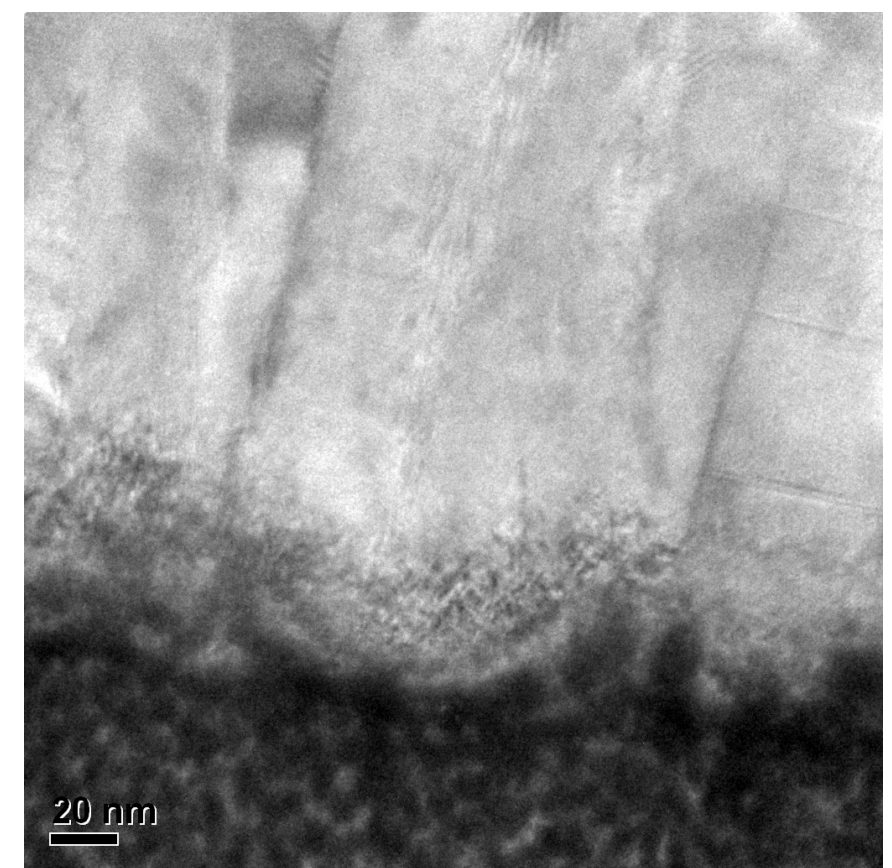

Fig. 2. Crystal structure at final stage of $\mathrm{ZnO}$ crystal growth 